\title{
Stroboscopic High-Duty-Cycle GHz Time-Resolved Microscope: First Proof of a Picosecond Beam Operated at GHz Level on a $200 \mathrm{keV}$ Beamline
}

\author{
Ao Liu ${ }^{1}$, Chunguang Jing ${ }^{1}$, Alexei Kanareykin ${ }^{1}$, Yubin Zhao ${ }^{1}$, Bryan Reed ${ }^{2}$, Daniel Masiel ${ }^{2}$, Yimei Zhu ${ }^{3}$, \\ June W. Lau ${ }^{4}$ \\ 1. Euclid TechLabs, Bolingbrook, USA. \\ 2. Integrated Dynamic Electron Solutions, Pleasanton, USA. \\ 3. Department of Condensed Matter Physics and Materials Science, Brookhaven National Laboratory, \\ Upton, USA. \\ 4. Materials Science and Engineering Division, National Institute of Standards and Technology, \\ Gaithersburg, USA.
}

In an ultrafast TEM operated in stroboscopic mode (denoted as "stroboscopic UTEM" from this point on), reversible processes in the specimen may be recorded, as long as data containing the same part of a cycle are repeatedly collected and summed over an extended period of time. Laser-based stroboscopic UTEMs generally do not operate at frequencies higher than $0.1 \mathrm{GHz}$ due to laser recharge limitations and the heat deposition on the specimens. On the other hand, systems amenable to electromagnetic excitations can benefit from an electromagnetically driven system to provide pump and probe with substantially higher repetition rate and beam current than the presently available stroboscopic UTEM. The high repetition rate leads to a short amount of time required to accumulate a measurable signal.

In the paper by Qiu [1], the compact GHz pulsing device composed of two RF modulators and three magnetic quadrupoles was proposed and described in detail. The device, referred to as a "chopper" or a "pulser", was designed as a retrofit to some of the most common JEOL microscopes [2], such as the 2000 and 3000 series. The main concept and physical model of this $\mathrm{GHz}$ stroboscopic pump-probe UTEM was presented by Baryshev et. al., at M\&M 2017.

The chopping module, which consists of a customized condenser ( $\mathrm{C} 0)$ and the chopper described above, is inserted between the gun and the microscope's first condenser lens. The customized C0 can produce a beam with a broad range of diameters and focal lengths along the optic axis. The two RF components modulate and then demodulate the beam transversely, while a chopping aperture in between them selects (chops) parts of the modulated beam and produces picosecond or sub-picosecond pulse trains at continuously tunable $\mathrm{GHz}$ frequencies. Excitation to the specimen is simultaneously delivered by the main RF generator, which also controls the pulsers. We use this RF signal as the means to synchronize the probe and the pump with controllable delays.

Fabrications of all components is now complete. At Euclid's R\&D facility, a $200 \mathrm{keV}$ electron gun from a JEOL 2000EX TEM was installed directly upstream of the $\mathrm{C} 0$ and the chopping module, in order to demonstrate their combined capabilities. Characterization of the $\mathrm{C} 0$ was performed by measuring the beam diameter as a function of distance from the source. The same detector setup also recorded the first demonstration of 10 ps pulse trains at arbitrary frequencies between 1 and $10 \mathrm{GHz}[2,3]$.

References:

[1] J Qiu et al, Ultramicroscopy 161 (2016), p. 130. 
[2] Certain commercial equipment, instruments, or materials are identified in this paper in order to specify the experimental procedure adequately. Such identification is not intended to imply recommendation or endorsement by the National Institute of Standards and Technology, nor is it intended to imply that the materials or equipment identified are necessarily the best available for the purpose.

[3] We are grateful for the crucial advice and assistance from Hideaki Arima, Bob Pohorenec, John Guerin, and many others at (JEOL USA). This work was supported by DOE BES SBIR program Grant no. DE-SC0013121, and NIST award SB1341-16-CN-0035.
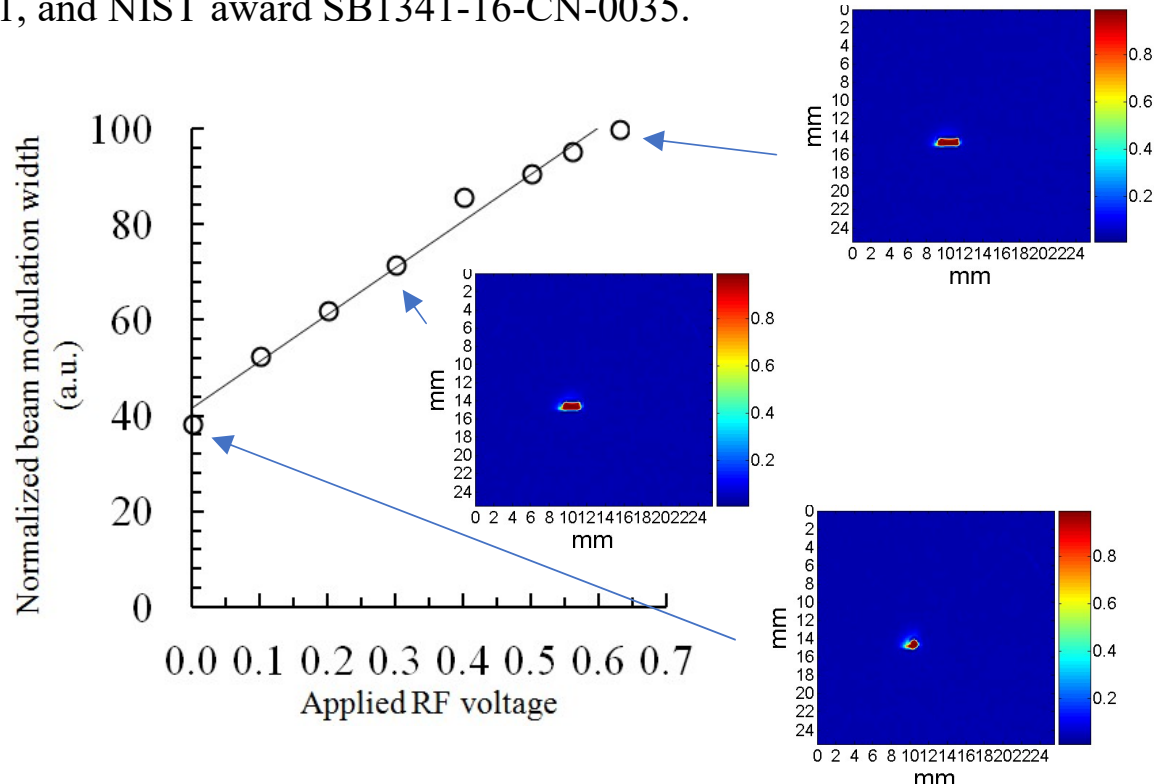

Figure 1. With the demodulator operating in the "enhanced modulation" mode, the transverse elongation is an accurate proxy for the magnitude of RF modulation, which is inversely proportional to the temporal resolution. Our data shows a linear dependence of the beam elongation width with the applied RF voltage.
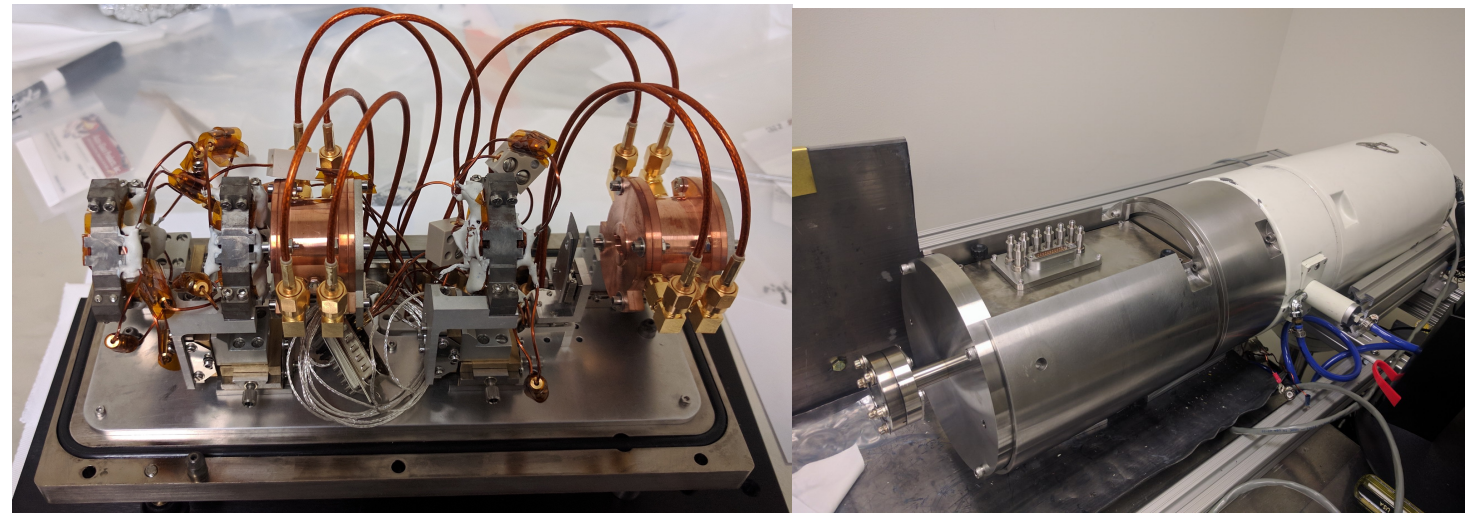

Figure 2. (left) Chopper components assembled on the mounting plate; (right) Customized C0 and chopper installed downstream the JEOL 2000EX gun. 